\title{
Editorial: Evolution and Biodiversity of Wild Polyploids
}

\author{
Elvira Hörandl ${ }^{1 *}$, Christoph Oberprieler ${ }^{2}$, Karol Marhold $^{3,4}$ and Natascha D. Wagner ${ }^{1}$ \\ ${ }^{1}$ Department of Systematics, Biodiversity and Evolution of Plants, University of Göttingen, Göttingen, Germany, ${ }^{2}$ Institute of \\ Plant Sciences, Evolutionary and Systematic Botany, University of Regensburg, Regensburg, Germany, ${ }^{3}$ Institute of Botany, \\ Plant Science and Biodiversity Centre, Slovak Academy of Sciences, Bratislava, Slovakia, ${ }^{4}$ Department of Botany, Faculty of \\ Science, Charles University, Prague, Czechia
}

Keywords: polyploidy, evolution, biogeography, biodiversity, repeatomes, transcriptomes

\section{Editorial on the Research Topic}

\section{Evolution and Biodiversity of Wild Polyploids}

Polyploidy is a major driver of evolution in plants. Angiosperms have undergone several polyploidization events in their evolutionary history (Leebens-Mack et al., 2019, Van De Peer et al., 2017) and polyploidy is an important factor causing speciation and influencing diversification of

OPEN ACCESS

Edited by: Marcial Escudero,

Sevilla University, Spain

Reviewed by:

João Loureiro,

University of Coimbra, Portugal

Yves Van de Peer,

Ghent University, Belgium

Martin A. Lysak,

Masaryk University, Czechia

*Correspondence:

Elvira Hörandl

elvira.hoerandl@

biologie.uni-goettingen.de

Specialty section:

This article was submitted to Plant Systematics and Evolution,

a section of the journal

Frontiers in Plant Science

Received: 10 June 2021

Accepted: 23 June 2021

Published: 20 July 2021

Citation:

Hörandl E, Oberprieler C, Marhold K and Wagner ND (2021) Editorial:

Evolution and Biodiversity of Wild

Polyploids.

Front. Plant Sci. 12:723439.

doi: 10.3389/fpls.2021.723439 flowering plants (Abbott et al., 2013, Soltis et al., 2014b). However, the processes of evolutionary origin, establishment, speciation and post-origin evolution of polyploid plants are still not wellunderstood. Furthermore, most research on polyploids is performed on few model taxa or on crop plants. Overcoming the gap between cultivated model organisms and natural systems is crucial for a complete understanding of polyploid evolution. Here we present a symposium on polyploid evolution in wild plants including 10 case studies that can be grouped around three major themes: first, origins and evolutionary histories of polyploids; second, aspects of genome evolution in polyploids; and third, the relationships between polyploidy and biogeography and ecology.

Natural origins of polyploid species, their phylogenetic relationships, and the impact of polyploidy on diversification are major topics of biodiversity research. Melichárková et al. demonstrate multiple and recurrent origins of autopolyploids and inter-cytotype matings in the genus Cardamine. This neopolyploid complex diversified further via allopatry and ecological differentiation. Reconstructing phylogenies of genera including polyploids have been notoriously difficult due to the frequent origin of polyploid species via hybridization (allopolyploidy). In the genus Salix, Wagner et al. elucidate multiple polyploidization events and post-origin genome evolution of mesopolyploid species by using phylogenomic approaches. Their results supported allopolyploid origins and their establishment as distinct species. Paule et al. reconstruct genome size evolution in the big plant family Bromeliaceae based on the phylogeny of this family. Results suggest that polyploidy and dysploidy have played a role in the survival of early diverged lineages under extreme climatic conditions, but did not enhance net diversification. Finally, Meudt et al. analyze polyploid frequencies in floras of oceanic islands and use phylogenetic path analysis to estimate effects of polyploidy on diversification. The results suggest that endemic diversification and speciation of the analyzed island floras were shaped by polyploidy in many cases. These papers seem to support also the general pattern that young polyploid complexes tend more to increase diversification rates than older ones (Landis et al., 2018).

The evolution of polyploid genomes is highly dynamic (Comai, 2005; Chen, 2007), but many molecular consequences of whole genome duplication are still not well-understood. Zagorski et al. analyze the repeatome of Hieracium species and their synthetic F1 hybrids by NGS analyses and FISH. They find a marked influence of hybridization on satellite and rDNA dynamics, whereas transposable elements showed no significant bursts but rather dosage-dependent patterns. 
Analyses of the repeatome in the mesopolyploids of Heliophila by Dogan et al. reveal a highly dynamic evolution of retrotransposons and tandem repeats, reflecting the post-origin cladogenesis in the genus. Boatwright et al. analyze gene expression patterns of homoeologs in mesopolyploid populations of Tragopogon compared to their parental species and reveal similar and convergent expression changes, but also stochastic silencing of loci. Moreover, the authors found significantly more non-additive expression in mesopolyploids compared to recently formed neopolyploids.

Polyploids are thought to have advantages for ecological niche shifts (Marchant et al., 2016, Soltis et al., 2014b) and for range expansions, specifically to more northern regions (Rice et al., 2019). Buono et al. conducted phylogeographical analyses, niche modeling and crossing experiments on two European polyploid Veronica species and found that despite some introgression species boundaries are maintained by spatial and ecological isolation. Duchoslav et al. analyze the niche dynamics of various cytotypes of Allium oleraceum and find niche expansions in tetraploids, but not in higher ploidy levels. High ploidies rather showed unfilled niches and a tendency toward synanthropic habitats. Spoelhof et al. use a modeling approach to understand the establishment of polyploids in natural habitats by including spatial factors and relationships between organisms, habitat shape, and population density. Results suggest that narrow, constrained habitats such as roadsides and coastlines may enhance polyploid establishment.

\section{REFERENCES}

Abbott, R., Albach, D., Ansell, S., Arntzen, J. W., Baird, S. J. E., Bierne, N., et al. (2013). Hybridization and speciation. J. Evol. Biol. 26, 229-246. doi: 10.1111/j.1420-9101.2012.02599.x

Chen, Z. J. (2007). Genetic and epigenetic mechanisms for gene expression and phenotypic variation in plant polyploids. Ann. Rev. Plant Biol. 58, 377-406. doi: 10.1146/annurev.arplant.58.032806.1 03835

Comai, L. (2005). The advantages and disadvantages of being polyploid. Nat. Rev. Genet. 6, 836-846. doi: 10.1038/nrg1711

Landis, J. B., Soltis, D. E., Li, Z., Marx, H. E., Barker, M. S., Tank, D. C., et al. (2018). Impact of whole-genome duplication events on diversification rates in angiosperms. Am. J. Bot. 105, 348-363. doi: 10.1002/ajb2.1060

Leebens-Mack, J. H., Barker, M. S., Carpenter, E. J., Deyholos, M. K., Gitzendanner, M. A., Graham, S. W., et al. (2019). One thousand plant transcriptomes and the phylogenomics of green plants. Nature 574:679. doi: 10.1038/s41586-0191693-2

Marchant, D. B., Soltis, D. E., and Soltis, P. S. (2016). Patterns of abiotic niche shifts in allopolyploids relative to their progenitors. New Phytol. 212, 708-718. doi: 10.1111/nph. 14069

Mayrose, I., Zhan, S. H., Rothfels, C. J., Magnuson-Ford, K., Barker, M. S., Rieseberg, L. H., et al. (2011). Recently formed polyploid plants diversify at lower rates. Science 333, 1257-1257. doi: 10.1126/science.1207205
The symposium highlights the diversity of evolutionary processes that are connected with polyploidy. Several papers point at the importance of post-origin evolution and diversification of polyploids, a topic that is hitherto been neglected in biodiversity research. We are at the infancies of understanding genome evolution and functionality in natural polyploid model systems. Here we present insights into repeatome evolution and gene expression studies. Whole genome sequencing and building reference genomes for wild model systems are highly needed to understand the consequences of polyploidization on genome structure and function. The symposium further shows various biogeographical and ecological scenarios for polyploids. Altogether, the case studies in this symposium refute the idea that polyploidization would be a dead end of evolution, as proposed by some researchers based on simple diversification statistics (Mayrose et al., 2011). The bias in such studies by undersampling, by neglecting reticulate evolution and different ages of diploids vs. polyploids was reviewed before (Soltis et al., 2014a). Careful case studies as presented here will provide a better understanding of the realized ecological and evolutionary potentials of polyploids.

\section{AUTHOR CONTRIBUTIONS}

EH wrote the Editorial with contributions from all co-authors. All authors contributed to the article and approved the submitted version.

Rice, A., Smarda, P., Novosolov, M., Drori, M., Glick, L., Sabath, N., et al. (2019). The global biogeography of polyploid plants. Nat. Ecol. Evol. 3:265. doi: 10.1038/s41559-018-0787-9

Soltis, D. E., Segovia-Salcedo, M. C., Jordon-Thaden, I., Majure, L., Miles, N. M., Mavrodiev, E. V., et al. (2014a). Are polyploids really evolutionary deadends (again)? A critical reappraisal of Mayrose et al. (2011). New Phytol. 202, 1105-1117. doi: 10.1111/nph.12756

Soltis, D. E., Visger, C. J., and Soltis, P. S. (2014b). The polyploidy revolution then... and now: Stebbins revisited. Am. J. Bot. 101, 1057-1078. doi: 10.3732/ajb.1400178

Van De Peer, Y., Mizrachi, E., and Marchal, K. (2017). The evolutionary significance of polyploidy. Nat. Rev. Genet. 18, 411-424. doi: $10.1038 / \mathrm{nrg} .2017 .26$

Conflict of Interest: The authors declare that the research was conducted in the absence of any commercial or financial relationships that could be construed as a potential conflict of interest.

Copyright (c) 2021 Hörandl, Oberprieler, Marhold and Wagner. This is an openaccess article distributed under the terms of the Creative Commons Attribution License (CC BY). The use, distribution or reproduction in other forums is permitted, provided the original author(s) and the copyright owner(s) are credited and that the original publication in this journal is cited, in accordance with accepted academic practice. No use, distribution or reproduction is permitted which does not comply with these terms. 